\title{
The initial Lorentz factors of fireballs inferred from the early X-ray data of SWIFT GRBs
}

\author{
R.-R. Xue ${ }^{1,2}$, Y.-Z. Fan ${ }^{3,1}$, and D.-M. Wei ${ }^{1,4}$ \\ ${ }^{1}$ Purple Mountain Observatory, Chinese Academy of Sciences, Nanjing 210008, PR China \\ e-mail: yizhong@nbi.dk \\ 2 Graduate School, Chinese Academy of Sciences, Beijing, 100012, PR China \\ 3 Niels Bohr International Academy, Niels Bohr Institute, University of Copenhagen, Blegdamsvej 17, 2100 Copenhagen, Denmark \\ 4 Joint Center for Particle Nuclear Physics and Cosmology of Purple Mountain Observatory - Nanjing University, Nanjing 210008, \\ PR China
}

Received 17 November 2008 / Accepted 10 February 2009

\begin{abstract}
Aims. We intend to determine the type of circumburst medium and directly measure the initial Lorentz factor $\Gamma_{0}$ of GRB outflows. Methods. If the early X-ray afterglow light curve has a peak and the whole profile across the peak is consistent with the standard external shock model, the early rise profile of light curves can be used to differentiate whether the burst was born in the interstellar medium (ISM) or in a stellar wind. In the thin shell case related to a subrelativistic reverse shock, the peak time occurring after the end of the prompt emission can be used to derive an accurate $\Gamma_{0}$, especially for the ISM case. The afterglow light curves for a flat electron spectrum $1<p<2$ have been derived analytically.

Results. In our GRB sample, we obtained $\Gamma_{0} \sim 300$ for the bursts born in ISM. We did not find any good case for bursts born in stellar wind and behaving as a thin shell that can be used to constrain $\Gamma_{0}$ reliably.
\end{abstract}

Key words. gamma rays: bursts - ISM: jets and outflows - radiation mechanisms: non-thermal

\section{Introduction}

Gamma-ray bursters are among the most mysterious celestial objects that have interested people since its first detection in 1967 (Klebesadel et al. 1973). The time variability of pulses, as short as a millisecond, limits this event to an object on a stellar scale. The random occurrence and the short time-duration of this kind of event has led to difficulties in detection. The dark era of research on gamma-ray bursts (GRBs) lasted until the release of X-ray afterglow data of GRB 970228, confirming GRBs at the cosmological distances (Costa et al. 1997).

The power-law decay of multi-waveband afterglows of many GRBs are consistent with the standard external shock model (Waxman 1997; Wijers et al. 1997). However, the multiwaveband afterglows were usually monitored several hours after the burst trigger before Swift era. The late afterglow, independent of the initial values of the fireball, cannot provide information about the fireball characteristics. X-ray Telescope (XRT) and Ultra-Violet Telescope (UVOT) onboard the Swift satellite (Gehrels et al. 2004) and other ground-based telescopes can slew to GRB within ten seconds and then begin observations. The early afterglow data released in Swift era provide us an opportunity to study properties of fireballs, e.g., the initial Lorentz factor of the fireball.

The fireball is expected to be a highly relativistic ejection from the central engine to avoid the "compact problem" (Shemi \& Piran 1990; Lithwick \& Sari 2001). After the radiationdominated acceleration phase, the fireball goes into a matterdominated phase when the fireball is no long accelerated.
The fireball keeps an approximately invariable velocity until it sweeps up a considerable mass of ambient medium. We call this episode the coasting phase (Piran et al. 1993). Although the profile of the early afterglow appears quite different from burst to burst and also from the X-ray to infrared bands, the peaks in the early afterglow light curves may indicate the arrival of the deceleration radius $\left(R_{\mathrm{d}}\right)$ in some GRBs. For example, Molinari et al. (2007) attributed the peaks in near-infrared afterglows of GRB 060418 and GRB 060607A to the end of the coasting phase and determined the initial Lorentz factors of the fireballs (see also Jin \& Fan 2007). Recently, Oates et al. (2009) have analyzed the early afterglows of Swift-UVOT data and measured the initial Lorentz factor of GRBs for those showing an early powerlaw increase in flux.

In contrast to these works, we now use the early X-ray data of Swift GRBs to constrain the initial Lorentz factors $\left(\Gamma_{0}\right)$. As a probe of $\Gamma_{0}$, the X-ray data is better than the optical data for the following reasons. (1) In the standard fireball model, the X-ray emission quickly decays with time after the outflow has decelerated, independent of the profile of the medium surrounding the progenitor (Fan \& Wei 2005). This occurs because both the typical frequencies of the forward shock (FS) and the reverse shock (RS) emission are usually below the X-ray band (see Table 1 for the light curves). The optical emission, however, will increase until the typical synchrotron frequency of the FS drops below the optical band (Sari et al. 1998). (2) In the thin shell case of interest, usually the RS X-ray emission is not strong enough to outshine the FS emission component. The origin of the X-ray peak can thus be reliably established. (3) X-ray afterglows are 
hardly influenced by the self-absorption effect and dust extinction, different from the emission at lower frequencies. One disadvantage of our method is that the early X-ray emission of most GRBs have been polluted by the delayed flares (Falcone et al. 2007) powered by the prolonged activity of the central engine. Fortunately, the X-ray flares usually have a decline as steep as $t^{-(3 \sim 10)}$, which is significantly sharper than what the fireball model predicts, so one can distinguish the peak in FS emission from the peak of the flare in the X-ray band convincingly.

\section{The early X-ray afterglow emission}

A very bright optical flash has been detected in GRB 990123 (Akerlof et al. 1999). The most widely discussed interpretation is the external RS model (Sari \& Piran 1999; Mészáros \& Rees 1999 however, it can also be produced by the internal shock model; e.g. Wei 2007). Since then, the RS emission in the optical band has been extensively investigated (see Zhang 2007, for a review). However, the RS X-ray emission has just been calculated by a few authors (Fan \& Wei 2005; Zou et al. 2005). In this work we focus on the profile of early X-ray afterglow light curves in different cases.

In the Fermi acceleration process, the power-law index of shocked electrons $p>2$ is the result (Gallant 2002). It has been taken as the standard scenario and widely used in the afterglow calculation (Sari et al. 1998; Chevalier \& Li 2000). Some afterglow modeling (Bhattacharya 2001; Dai \& Cheng 2001), however, favor a flat electron spectrum $1<p<2$, for which a reliable estimate of the afterglow emission is still unavailable. We discuss such a scenario in some detail in Sect. 2.2.

\subsection{The case of $p>2$}

First, we discuss the "thin shell case" for which $t_{\times}>T_{90}$, where $t_{\times}$is the crossing time of RS and $T_{90}$ is the duration of the burst, referring to a subrelativistic RS. Assuming typical parameters (e.g., the fraction of the shock energy given to the electrons $\epsilon_{\mathrm{e}}=0.1$, the fraction given to the magnetic field $\epsilon_{B}=0.01$, the total energy of the fireball $E=10^{52} \mathrm{erg}$ and $p=2.3$ ), we have the typical synchrotron radiation frequency and the cooling frequency of the FS emission $v_{\mathrm{m}}^{\mathrm{f}}=2 \times 10^{16} \mathrm{~Hz}(t / 100 \mathrm{~s})^{-3 / 2}$, $v_{\mathrm{c}}^{\mathrm{f}}=8 \times 10^{16} \mathrm{~Hz} \mathrm{n}_{0}^{-1}(t / 100 \mathrm{~s})^{-1 / 2}$ for bursts born in ISM (Sari et al. 1998) and $v_{\mathrm{m}}^{\mathrm{f}}=5 \times 10^{16} \mathrm{~Hz}(t / 100 \mathrm{~s})^{-3 / 2}, v_{\mathrm{c}}^{\mathrm{f}}=4.8 \times$ $10^{12} \mathrm{~Hz}(t / 100 \mathrm{~s})^{1 / 2} A_{*}^{-2}$ born in the wind medium (Chevalier \& Li 2000), where $n$ and $A_{*}$ are two medium parameters: $n$ indicates the density of ISM, in units of $\mathrm{cm}^{-3}$, while $A_{*}$ is the dimensionless parameter of stellar wind environment. $t$ indicates the observed time postburst. The convention $Q_{x}=Q / 10^{x}$ has been adopted in this paper, in units of cgs.

Combined with $v_{\mathrm{m}}^{\mathrm{f}}\left(t_{\times}\right)>v_{\mathrm{m}}^{\mathrm{r}}\left(t_{\times}\right), v_{\mathrm{c}}^{\mathrm{f}}\left(t_{\times}\right) \approx v_{\mathrm{c}}^{\mathrm{r}}\left(t_{\times}\right)$, we find that both $v_{\mathrm{m}}$ and $v_{\mathrm{c}}$ of FS and RS emission (marked by the subscripts $f$ and $r$, respectively) are below the X-ray band $v_{\mathrm{X}} \sim$ $10^{17} \mathrm{~Hz}$, assuming typical parameters. If the shock parameters are similar for the FS and the RS, the flux contrast between the RS and FS X-ray emission is thus ${ }^{1}$ (Fan \& Wei 2005)

$\frac{F_{v_{\mathrm{X}}}^{\mathrm{r}}\left(t_{\times}\right)}{F_{v_{\mathrm{X}}}^{\mathrm{f}}\left(t_{\times}\right)} \approx \Gamma_{0}\left(\frac{\gamma_{34, \times}-1}{\Gamma_{\times}-1}\right)^{p-1}$,

\footnotetext{
1 This expression is valid in the "thin shell" case. In the "thick shell" case, such an expression is an upper limit since the total number of the RS electrons is less than $\Gamma_{0}$ times that of the FS electrons (see Eq. (3)).
}

where the subscript $\times$ represents the parameters measured at $t_{\times}$, and $\Gamma_{0}$ and $\Gamma_{\times}$represent the initial Lorentz factor and the instant Lorentz factor at $t_{\times}$, respectively. The corresponding relative Lorentz factor is $\gamma_{34, \times} \approx\left(\Gamma_{0} / \Gamma_{\times}+\Gamma_{\times} / \Gamma_{0}\right) / 2$.

In the so-called "thin shell case", $\Gamma_{\times} \approx \Gamma_{0} / 2$ and $\gamma_{34, \times} \approx$ 1.25. For a typical $p \sim 2.3$, Eq. (1) gives

$\frac{F_{v_{\mathrm{X}}}^{\mathrm{r}}\left(t_{\times}\right)}{F_{v_{\mathrm{X}}}^{\mathrm{f}}\left(t_{\times}\right)} \approx 2^{1-p} \Gamma_{0}^{2-p} \sim O(0.1)$,

for $\Gamma_{0} \sim$ a few $\times 100$, which suggests that the $R S X$-ray emission can be ignored. This conclusion is unchanged if $v_{\mathrm{m}}^{\mathrm{f}}$ is actually above $v_{\mathrm{X}}$, because in such a case the FS X-ray emission would be stronger.

In the "thick shell case" (for which $t_{\times} \sim T_{90}$ ), particularly for a relativistic RS satisfying $\gamma_{34, \times}-1 \approx \Gamma_{0} / 2 \Gamma_{\times}$, we have

$\frac{F_{v_{\mathrm{X}}}^{\mathrm{r}}\left(t_{\times}\right)}{F_{v_{\mathrm{X}}}^{\mathrm{f}}\left(t_{\times}\right)}<2\left(\gamma_{34, \times}-1\right)^{\mathrm{p}} \Gamma_{\times}^{2-p}$.

As a result, the RS X-ray emission may be able to outshine the FS component.

Thin shell case: the RS X-ray emission is unimportant, so the afterglow is dominated by the FS component. The bulk Lorentz factor $\Gamma$ is nearly a constant in this coasting phase $\left(t<t_{\times}\right)$. We then have $v_{\mathrm{m}}^{\mathrm{f}} \propto t^{-k / 2}, v_{\mathrm{c}}^{\mathrm{f}} \propto t^{3 k / 2-2}$, and the maximal specific flux $F_{v, \text { max }}^{\mathrm{f}} \propto t^{3(1-k / 2)}(k=0$ for ISM and $k=2$ for the stellar wind $)$. As a result, the FS X-ray emission evolves as $F_{v_{X}}^{\mathrm{f}} \propto t^{2-(2+p) k / 4}$ with time. In the case of ISM, $F_{v_{\mathrm{X}}}^{\mathrm{f}} \propto t^{2}$ increase quickly with time, while in the wind case, $F_{v_{\mathrm{X}}}^{\mathrm{f}} \propto t^{(2-p) / 2}$ decrease slowly with time.

Thick shell case: in the case of ISM, we have $\Gamma \propto t^{-1 / 4}$, $v_{\mathrm{m}}^{\mathrm{r}} \propto t^{0}, v_{\mathrm{c}}^{\mathrm{r}} \propto t^{-1}, F_{v, \max }^{\mathrm{r}} \propto t^{1 / 2}$, and then derive $F_{v_{\mathrm{X}}}^{\mathrm{r}} \propto t^{0}$ (see also Kobayashi 2000) when $t<t_{\times}$. The simultaneous FS X-ray emission is $F_{v_{\mathrm{X}}}^{\mathrm{f}} \propto t^{(2-p) / 2}$. In the wind case, both the FS and the RS emission decrease slowly with time as $t^{(2-p) / 2}$ when $t<t_{\times}$ (see also Fan \& Wei 2005).

For $t>t_{\times}$, it is well known that $F_{v_{\mathrm{X}}}^{\mathrm{f}} \propto t^{(2-3 p) / 4}$, independent of the type of circumburst medium for both thin shell and thick shell cases.

Please note that we assume in our above analysis that both $v_{\mathrm{m}}$ and $v_{\mathrm{c}}$ of FS and RS are well below the XRT band. More general results have been summarized in Table 1. One can see that $F_{v_{X}}^{\mathrm{f}} \propto$ $t^{-1 / 4}$ is also possible but only for a fast-cooling forward shock. Its spectrum should be $F_{v} \propto v^{-1 / 2}$, which can be distinguished from the shallow decline predicted for the FS and RS emission in the wind case at time $t<t_{\mathrm{p}}$.

\subsection{The case of $1<p<2$}

With the shock jump conditions, Bhattacharya (2001) derived a minimum Lorentz factor $\left(\gamma_{\mathrm{m}}\right)$ of the electrons that depends on the maximal one $\left(\gamma_{\mathrm{M}}\right)$, i.e., $\gamma_{\mathrm{m}} \approx\left\{(2-p) m_{\mathrm{p}} \epsilon_{\mathrm{e}} \Gamma_{\mathrm{sh}} \gamma_{\mathrm{M}}^{p-2} /[(p-\right.$ 1) $\left.\left.m_{\mathrm{e}}\right]\right\}^{1 /(p-1)}$, where $\Gamma_{\mathrm{sh}}$ is the Lorentz factor of the shock, $m_{\mathrm{p}}$ and $m_{\mathrm{e}}$ are the rest mass of protons and electrons, respectively. However, in reality, particle acceleration proceeds from low to high energy. Both $\gamma_{\mathrm{m}}$ and $\gamma_{\mathrm{M}}$ should be determined by the first shock-crossing and by radiative losses or escape from 
Table 1. The temporal behavior of the X-ray afterglow light curves in the case of $p>2$.

\begin{tabular}{llll}
\hline \hline & Emission regime & ISM & Wind \\
\hline Thin shell case (FS) & $v_{\mathrm{X}}>\max \left\{v_{\mathrm{c}}^{\mathrm{f}}, v_{\mathrm{m}}^{\mathrm{f}}\right\}$ & $t^{2}$ & $t^{(2-p) / 2}$ \\
$t<t_{\mathrm{p}}$ & $v_{\mathrm{c}}^{\mathrm{f}}<v_{\mathrm{X}}<v_{\mathrm{m}}^{\mathrm{f}}$ & $t^{2}$ & $t^{1 / 2}$ \\
$t<t_{\mathrm{p}}$ & $v_{\mathrm{m}}^{\mathrm{f}}<v_{\mathrm{X}}<v_{\mathrm{c}}^{\mathrm{f}}$ & $t^{3}$ & $t^{-(p-1) / 2}$ \\
$t<t_{\mathrm{p}}$ & $v_{\mathrm{m}}^{\mathrm{r}}<v_{\mathrm{c}}^{\mathrm{r}}<v_{\mathrm{X}}$ & $t^{2 p-1}$ & $t^{-(p-2) / 2}$ \\
\hline Thin shell case (RS) & $v_{\mathrm{m}}^{\mathrm{r}}<v_{\mathrm{X}}<v_{\mathrm{c}}^{\mathrm{r}}$ & $t^{2 p}$ & $t^{-(p-1) / 2}$ \\
$t<t_{\mathrm{p}}$ & & & \\
$t<t_{\mathrm{p}}$ & $v_{\mathrm{X}}>\max \left\{v_{\mathrm{c}}^{\mathrm{r}}, v_{\mathrm{m}}^{\mathrm{r}}\right\}$ & $t^{0}$ & $t^{(2-p) / 2}$ \\
\hline Thick shell case (RS) & $v_{\mathrm{c}}^{\mathrm{r}}<v_{\mathrm{X}}<v_{\mathrm{m}}^{\mathrm{r}}$ & $t^{0}$ & $t^{1 / 2}$ \\
$t<t_{\mathrm{p}}$ & $v_{\mathrm{m}}^{\mathrm{r}}<v_{\mathrm{X}}<v_{\mathrm{c}}^{\mathrm{r}}$ & $t^{1 / 2}$ & $t^{-(p-1) / 2}$ \\
$t<t_{\mathrm{p}}$ & $v_{\mathrm{X}}>\max \left\{v_{\mathrm{c}}^{\mathrm{f}}, v_{\mathrm{m}}^{\mathrm{f}}\right\}$ & $t^{(2-3 p) / 4}$ & $t^{(2-3 p) / 4}$ \\
$t<t_{\mathrm{p}}$ & $v_{\mathrm{c}}^{\mathrm{f}}<v_{\mathrm{X}}<v_{\mathrm{m}}^{\mathrm{f}}$ & $t^{-1 / 4}$ & $t^{-1 / 4}$ \\
\hline Late FS emission & $v_{\mathrm{m}}^{\mathrm{f}}<v_{\mathrm{X}}<v_{\mathrm{c}}^{\mathrm{f}}$ & $t^{3(1-p) / 4}$ & $t^{(1-3 p) / 4}$ \\
$t>t_{\mathrm{p}}$ & & & \\
$t>t_{\mathrm{p}}$ & $v_{\mathrm{X}}>\max \left\{v_{\mathrm{c}}^{\mathrm{f}}, v_{\mathrm{m}}^{\mathrm{f}}\right\}$ & $t^{-p}$ & $t^{-p}$ \\
$t>t_{\mathrm{p}}$ & $v_{\mathrm{c}}^{\mathrm{f}}<v_{\mathrm{X}}<v_{\mathrm{m}}^{\mathrm{f}}$ & $t^{-1}$ & $t^{-1}$ \\
\hline Late FS emission (jet) & $v_{\mathrm{m}}^{\mathrm{f}}<v_{\mathrm{X}}<v_{\mathrm{c}}^{\mathrm{f}}$ & $t^{-p}$ & $t^{-p}$ \\
$t>t_{\mathrm{j}}$ & & \\
$t>t_{\mathrm{j}}$ & &
\end{tabular}

Note. Observationally the crossing time $t_{\times}$marks the beginning of the late sharp decline, and we denote such a timescale by $t_{\mathrm{p}}$. $t_{\mathrm{j}}$ is the jet break time.

the acceleration region, respectively; hence, $\gamma_{\mathrm{m}}$ should have no "knowledge" of $\gamma_{\mathrm{M}}$ (Panaitescu 2006, private communication). Motivated by the above arguments, we assume in this work that $\gamma_{\mathrm{m}} \approx\left(\epsilon_{\mathrm{e}} / \mathrm{f}(p)\right)\left(\Gamma_{\mathrm{sh}}-1\right) m_{\mathrm{p}} / m_{\mathrm{e}}$, where $f(p)$ is a function of $p$. Such treatment requires that only a small fraction $\mathcal{R}$ of the upstream material has been accelerated; otherwise, the energy momentum conservation law will be violated.

Assuming that the shock-accelerated electrons have a powerlaw energy distribution $\mathrm{d} n / \mathrm{d} \gamma_{\mathrm{e}} \propto\left(\gamma_{\mathrm{e}}-1\right)^{-p}$ for $\gamma_{\mathrm{m}} \leq \gamma_{\mathrm{e}} \leq \gamma_{\mathrm{M}}$, with the shock jump conditions that $\int_{\gamma_{\mathrm{m}}}^{\gamma_{\mathrm{M}}}\left(\mathrm{d} n / \mathrm{d} \gamma_{\mathrm{e}}\right) \mathrm{d} \gamma_{\mathrm{e}}=4 \mathcal{R} \Gamma_{\mathrm{sh}} n_{\mathrm{u}}$ and $\int_{\gamma_{\mathrm{m}}}^{\gamma_{\mathrm{M}}}\left(\gamma_{\mathrm{e}}-1\right) m_{\mathrm{e}} c^{2}\left(\mathrm{~d} n / \mathrm{d} \gamma_{\mathrm{e}}\right) \mathrm{d} \gamma_{\mathrm{e}}=4 \Gamma_{\mathrm{sh}}\left(\Gamma_{\mathrm{sh}}-1\right) \epsilon_{\mathrm{e}} n_{\mathrm{u}} m_{\mathrm{p}} c^{2}$, we have

$$
\begin{aligned}
\mathcal{R} & \approx \frac{2-p}{p-1} \epsilon_{\mathrm{e}}\left(\frac{\epsilon_{\mathrm{e}}}{f(p)}\right)^{1-p}\left(\frac{m_{\mathrm{p}}}{m_{\mathrm{e}}}\right)^{2-p}\left(\Gamma_{\mathrm{sh}}-1\right)^{2-p} \gamma_{\mathrm{M}}^{p-2} \\
& \propto\left(\Gamma_{\mathrm{sh}}-1\right)^{2-p} B^{1-p / 2} .
\end{aligned}
$$

We also assume that the maximal Lorentz factor is limited by the synchrotron losses and is given by (Cheng \& Wei 1996)

$\gamma_{\mathrm{M}} \approx 4 \times 10^{7} B^{-1 / 2}$,

where $B=\sqrt{32 \pi \varepsilon_{B} \Gamma_{\mathrm{sh}}\left(\Gamma_{\mathrm{sh}}-1\right) n_{\mathrm{u}} m_{\mathrm{p}} c^{2}}$ is the magnetic field strength of the shock region, $n_{\mathrm{u}}$ the number density of the upstream medium measured in its rest frame, and $c$ the speed velocity of light. The afterglow light curves in the case of $1<p<2$ are different from those presented in Table 1 by a factor of $\mathcal{R}$ given below.

Assuming the $\mathrm{X}$-band is above $\max \left\{v_{\mathrm{c}}^{\mathrm{r}}, v_{\mathrm{m}}^{\mathrm{r}}, v_{\mathrm{c}}^{\mathrm{f}}, v_{\mathrm{m}}^{\mathrm{f}}\right\}$, the flux contrast between the RS and the FS emission can be estimated (for a thin shell) by

$\frac{F_{v_{\mathrm{x}}}^{\mathrm{r}}\left(t_{\times}\right)}{F_{v_{\mathrm{x}}}^{\mathrm{f}}\left(t_{\times}\right)} \approx \Gamma_{0}\left(\frac{\gamma_{34, \times}-1}{\Gamma_{\times}-1}\right)^{p-1}\left(\frac{\gamma_{34, \times}-1}{\Gamma_{\times}-1}\right)^{2-p} \sim \frac{1}{2}$.
Table 2. Temporal behavior of the X-ray afterglow light curves in the

\begin{tabular}{|c|c|c|c|}
\hline & Emission regime & ISM & Wind \\
\hline $\begin{array}{l}\text { Thin shell case (FS) } \\
t<t_{\mathrm{p}} \\
t<t_{\mathrm{p}} \\
t<t_{\mathrm{p}}\end{array}$ & $\begin{array}{l}v_{\mathrm{X}}>\max \left\{v_{\mathrm{c}}^{\mathrm{f}}, v_{\mathrm{m}}^{\mathrm{f}}\right\} \\
v_{\mathrm{c}}^{\mathrm{f}}<v_{\mathrm{X}}<v_{\mathrm{m}}^{\mathrm{f}} \\
v_{\mathrm{m}}^{\mathrm{f}}<v_{\mathrm{X}}<v_{\mathrm{c}}^{\mathrm{f}}\end{array}$ & $\begin{array}{l}t^{2} \\
t^{2} \\
t^{3}\end{array}$ & $\begin{array}{l}t^{0} \\
t^{(p-1) / 2} \\
t^{-1 / 2} \\
\end{array}$ \\
\hline $\begin{array}{l}\text { Thin shell case (RS) } \\
t<t_{\mathrm{p}} \\
t<t_{\mathrm{p}}\end{array}$ & $\begin{array}{l}v_{\mathrm{m}}^{\mathrm{r}}<v_{\mathrm{c}}^{\mathrm{r}}<v_{\mathrm{X}} \\
v_{\mathrm{m}}^{\mathrm{r}}<v_{\mathrm{X}}<v_{\mathrm{c}}^{\mathrm{r}}\end{array}$ & $\begin{array}{l}t^{3} \\
t^{4} \\
\end{array}$ & $\begin{array}{l}t^{0} \\
t^{-1 / 2} \\
\end{array}$ \\
\hline $\begin{array}{l}\text { Thick shell case }(\mathrm{RS}) \\
t<t_{\mathrm{p}} \\
t<t_{\mathrm{p}} \\
t<t_{\mathrm{p}}\end{array}$ & $\begin{array}{l}v_{\mathrm{X}}>\max \left\{v_{\mathrm{c}}^{\mathrm{r}}, v_{\mathrm{m}}^{\mathrm{r}}\right\} \\
v_{\mathrm{c}}^{\mathrm{r}}<v_{\mathrm{X}}<v_{\mathrm{m}}^{\mathrm{r}} \\
v_{\mathrm{m}}^{\mathrm{r}}<v_{\mathrm{X}}<v_{\mathrm{c}}^{\mathrm{r}}\end{array}$ & $\begin{array}{l}t^{-3(2-p) / 8} \\
t^{-3(2-p) / 8} \\
t^{(3 p-2) / 8}\end{array}$ & $\begin{array}{l}t^{0} \\
t^{(p-1) / 2} \\
t^{-1 / 2}\end{array}$ \\
\hline $\begin{array}{l}\text { Late FS emission } \\
t>t_{\mathrm{p}} \\
t>t_{\mathrm{p}} \\
t>t_{\mathrm{p}}\end{array}$ & $\begin{array}{l}v_{\mathrm{X}}>\max \left\{v_{\mathrm{c}}^{\mathrm{f}}, v_{\mathrm{m}}^{\mathrm{f}}\right\} \\
v_{\mathrm{c}}^{\mathrm{f}}<v_{\mathrm{X}}<v_{\mathrm{m}}^{\mathrm{f}} \\
v_{\mathrm{m}}^{\mathrm{f}}<v_{\mathrm{X}}<v_{\mathrm{c}}^{\mathrm{f}}\end{array}$ & $\begin{array}{l}t^{-(10+3 p) / 16} \\
t^{-(22-9 p) / 16} \\
t^{-(6+3 p) / 16}\end{array}$ & $\begin{array}{l}t^{-(6+p) / 8} \\
t^{-(12-5 p) / 8} \\
t^{-(8+p) / 8}\end{array}$ \\
\hline $\begin{array}{l}\text { Late FS emission (jet) } \\
t>t_{\mathrm{j}} \\
t>t_{\mathrm{j}} \\
t>t_{\mathrm{j}}\end{array}$ & $\begin{array}{l}v_{\mathrm{X}}>\max \left\{v_{\mathrm{c}}^{\mathrm{f}}, v_{\mathrm{m}}^{\mathrm{f}}\right\} \\
v_{\mathrm{c}}^{\mathrm{f}}<v_{\mathrm{X}}<v_{\mathrm{m}}^{\mathrm{f}} \\
v_{\mathrm{m}}^{\mathrm{f}}<v_{\mathrm{X}}<v_{\mathrm{c}}^{\mathrm{f}}\end{array}$ & $\begin{array}{l}t^{-(p+6) / 4} \\
t^{(3 p-10) / 4} \\
t^{-(p+6) / 4}\end{array}$ & $\begin{array}{l}t^{-(p+6) / 4} \\
t^{(3 p-10) / 4} \\
t^{-(p+6) / 4}\end{array}$ \\
\hline
\end{tabular}
case of $1<p<2$.

Note. Observationally the crossing time $t_{\times}$marks the beginning of the late sharp decline, denoting such a timescale by $t_{\mathrm{p}} . t_{\mathrm{j}}$ is the jet break time.

As a result, in the thin shell case, the RS X-ray emission is usually outshone by the FS X-ray radiation for both a flat electron spectrum $(1<p<2)$ and a standard electron spectrum $(p>2)$.

For the FS before deceleration, we have $\Gamma_{\mathrm{sh}} \propto t^{0}, B \propto$ $R^{-k / 2} \propto t^{-k / 2}$, and $\mathcal{R} \propto t^{-k(2-p) / 4}$. For the GRBs born in ISM in the case of a thin shell, we have $\Gamma_{\text {sh }}-1 \propto t^{2}$ (Fan et al. 2002), $B \propto t^{0}$, and $\mathcal{R} \propto t^{2(2-p)}$ for the RS. In the case of a thick shell, for the RS we have $\Gamma_{\text {sh }}-1 \propto t^{-1 / 4}$ (Kobayashi 2000), $B \propto t^{-(2+3 k) / 8}$, and $\mathcal{R} \propto t^{-(2-p)(6+k) / 16}$. For the GRBs born in the wind and for the RS we have $\Gamma_{\text {sh }}-1 \propto t^{0}, B \propto t^{-1}$, and $\mathcal{R} \propto t^{-(2-p) / 2}$.

For the FS taking a Blandford-McKee profile, we have $\Gamma_{\mathrm{sh}} \sim$ $\Gamma \propto R^{(k-3) / 2} \propto t^{(k-3) /(8-2 k)}, B \propto \Gamma_{\mathrm{sh}} R^{-k / 2} \propto R^{-3 / 2} \propto t^{-3 /(8-2 k)}$, and $\mathcal{R} \propto t^{\frac{(2 k-9)(2-p)}{4(4-k)}}$. As long as the edge of the ejecta is visible, the sideways expansion may be so strong that it cannot be ignored, and the dynamics is governed by $\Gamma \propto t^{-1 / 2}$ (Rhoads 1999). We then have $\mathcal{R} \propto t^{-3(2-p) / 4}$, independent of $k$. The detailed light curves are summarized in Table 2 .

\section{Case studies}

In the thick shell case, the RS disappears almost simultaneously with the prompt X-ray and $\gamma$-ray emission. As a result, the RS X-ray emission may be outshone by the prompt emission component. Moreover, for a thick shell, we need self-consistent modeling of the FS and RS emission to get $\gamma_{34, \times}$ and then $\Gamma_{0}$. In such a process, quite a few free parameters are introduced and the constraint on $\Gamma_{0}$ is uncertain. In this work, we only focus on the thin shell case with $t_{\mathrm{p}}=t_{\times}>T_{90}$.

As summarized in Tables 1 and 2, the outflow expanding into the ISM in the thin shell case will give rise to an increase no shallower than $t^{2}$, while expanding into the wind cannot account for an increase steeper than $t^{1 / 2}$. Therefore we can judge whether the GRB was born in the ISM or wind medium according to the sharpness of flux increase. One can also speculate that the $\mathrm{X}$-ray data for the GRBs born in the wind are not very good 


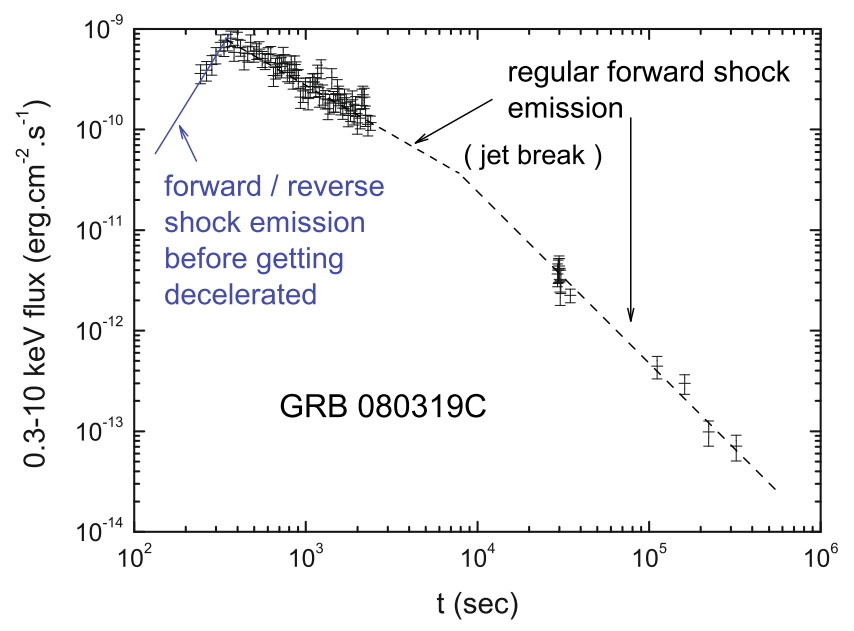

Fig. 1. X-ray afterglow light curve of GRB 080319C and its interpretation. The XRT data are from http://www.swift.ac.uk/ xrt_curves/00306778/ (Evans et al. 2007).

probes of the initial Lorentz factor of the ejecta due to the lack of a distinguished peak for typical parameters that give rise to $v_{\mathrm{X}}>\max \left\{v_{\mathrm{m}}, v_{\mathrm{c}}\right\}$.

The number of GRBs recorded by XRT exceeds 160 till August 1, 2008. Most of them, however, play no role in constraining $\Gamma_{0}$. For our purpose, the light curves have to be characterized by (i) a distinguished/single peak; (ii) across the peak, a smooth transition to a single power-law decay is followed and the whole profile must be consistent with the standard afterglow model (Sari et al. 1998; Chevalier \& Li 2000).

We checked all the Swift X-ray afterglows and find 7 GRBs whose early peaks may be attributed to the arrival of $R_{\mathrm{d}}$, and the post-peak temporal behaviors of these bursts are consistent with the standard afterglow model. All these bursts except GRB 051111 have the X-ray afterglow peaks much later than the end of the prompt emission and thus fall in the thin shell case. A good example is that of GRB 080319C (see Fig. 1). We divide our GRB sample, in total 6 events, into two groups: 4 were born in ISM and 2 that might be born in wind, based on the rise behavior of the early X-ray afterglow light curve.

\subsection{ISM case}

GRB 060801: the BAT light curve showed a short burst with a duration of about $0.5 \mathrm{~s}$. The candidate host galaxy has a redshift of 1.131 as given by Cucchiara et al. (2006). The XRT began taking data $63 \mathrm{~s}$ after the BAT trigger (Racusin et al. 2006a). The flux increased with time in the form of $\sim t^{2.3}$ and peaked $\sim 110 \mathrm{~s}$ after the BAT trigger, followed by a decay of $\sim t^{-1.19 \pm 0.16}$ with time to $\sim 400 \mathrm{~s}$ from the trigger (Racusin et al. 2006b). The XRT spectrum is $F_{v} \propto v^{-0.67 \pm 0.2}$. The decline and the spectrum are consistent with the fireball afterglow model, supposing that $p \sim 2.3$ and $v_{\mathrm{m}}^{\mathrm{f}}<v_{\mathrm{x}}<v_{\mathrm{c}}^{\mathrm{f}}$ (see Table 1). It is straightforward to show that, for $v_{\mathrm{m}}^{\mathrm{f}}<v_{\mathrm{X}}<v_{\mathrm{c}}^{\mathrm{f}}$, the FS X-ray emission still dominates the RS X-ray component.

GRB 060926: the peak occurred around $430 \mathrm{~s}$ after the BAT trigger. Before and after the peak, the flux evolved as $\sim t^{2.0}$ and $\sim t^{-1.4}$, respectively. Though Holland et al. (2006) considered this peak as a flare, the post-peak flux decayed temporally in the same way as the late time afterglows. D'Elia et al. (2006) claim a redshift of $z=3.20$ based on a damped Ly $\alpha$ line and various absorption features. The XRT spectrum is $F_{v} \propto v^{-1.1 \pm 0.2}$. The decline and the spectrum are consistent with the fireball afterglow model supposing that $p \sim 2.4$ and $v_{\mathrm{x}}>\max \left\{v_{\mathrm{c}}^{\mathrm{f}}, v_{\mathrm{m}}^{\mathrm{f}}\right\}$ (see Table 1).

GRB 080319C: the XRT began observing the burst 224 s after the BAT trigger (Pagani et al. 2008). The redshift $z=1.95$ was found from absorption lines of the afterglow spectrum (Wiersema et al. 2008). As shown in Fig. 1, a quick increase in flux $\sim t^{2.4}$ is obvious up to $\sim 360 \mathrm{~s}$ after the GRB trigger. After the peak time, the late afterglow exhibited a broken power law a relatively shallow decline $\sim t^{-0.86}$ followed by a $\sim t^{-1.87}$ profile. We attribute the late steep decline to the jet effect of this GRB. With a flat electron spectrum $p \sim 1.5$, both the temporal behavior of light curves in the two late episodes and the $\mathrm{X}$-ray spectrum $F_{v} \propto v^{-0.74 \pm 0.06}$ can be reproduced provided that $v_{\mathrm{x}}>\max \left\{v_{\mathrm{c}}^{\mathrm{f}}, v_{\mathrm{m}}^{\mathrm{f}}\right\}$ (see Table 2$)$.

GRB 080413B: the BAT light curve showed a single-peaked structure with a duration of about $3 \mathrm{~s}$ (Stamatikos et al. 2008b). The early XRT data recorded a steep increase in flux, peaking around $167 \mathrm{~s}$ from the trigger. The X-ray light curve is wellfitted by a simple power law, with a decay slope of $0.88 \pm 0.06$ from $167 \mathrm{~s}$ to $10^{4} \mathrm{~s}$ after the burst trigger (Troja \& Stamatikos 2008). Based on the detection of numerous absorption features, including Fe II, Mg II, and Mg I lines, Vreeswijk et al. (2008) infer a redshift $z=1.10$. The XRT spectrum is $F_{v} \propto v^{-1.05 \pm 0.1}$. The decline and the spectrum are roughly consistent with the fireball afterglow model, supposing that $p \sim 2$ and $v_{\mathrm{X}}>\max \left\{v_{\mathrm{c}}^{\mathrm{f}}, v_{\mathrm{m}}^{\mathrm{f}}\right\}$ (see Table 1).

\subsection{Wind case?}

GRB 080307: in the first orbit of the XRT data, the emission rose slowly, $\sim t^{0.6}$, peaking at $\sim 240 \mathrm{~s}$ after the BAT trigger, after which the decay can be modeled with a single power law with the temporal index $\alpha=1.83 \pm 0.08$ up to several ks after the burst trigger, although there was an abnormal flat in flux later (Page et al. 2008). The X-ray spectrum is $F_{v} \propto v^{-0.74 \pm 0.22}$. As shown in Table 1 in the thin shell case for $v_{\mathrm{c}}^{\mathrm{f}}<v_{\mathrm{x}}<v_{\mathrm{m}}^{\mathrm{f}}$, the FS emission of an ejecta expanding into the stellar wind can give rise to an initial rise $t^{0.5}$, roughly consistent with the data. However, this model gives a decline $t^{-1 / 4}$ after the X-ray peak, deviating from the data significantly, so this event won't be included in our $\Gamma_{0}$ constraint.

GRB 080409: the XRT began observing this burst 84 s after the BAT trigger. The light curve showed an initial increase with a power-law slope of $\sim 0.6$ and entered a peak at $\sim 509$ s after the burst onset. The light curve then turned over to decay with a power-law slope of $0.89 \pm 0.09$ (Holland et al. 2008). The XRT spectrum is $F_{v} \propto v^{-1.2 \pm 0.6}$. Similar to GRB 080307, an initial rise $t^{0.6}$ may be the FS emission of an ejecta born in wind. However, this model gives a decline $t^{-1 / 4}$ after the X-ray peak unless we see the edge of the ejecta for which the decline can be as steep as $t^{-1}$ (see Table 1). Again, we do not include it in the following $\Gamma_{0}$ constraint. 
Table 3. GRB sample.

\begin{tabular}{lccccccc}
\hline \hline GRB & $z^{a}$ & $E_{\gamma}\left(10^{52} \mathrm{erg}\right)^{b}$ & $T_{90}(\mathrm{~s})^{\mathrm{c}}$ & $t_{\mathrm{p}}(\mathrm{s})$ & medium type & $\Gamma_{0}^{d}$ & References \\
\hline 060801 & 1.131 & 0.55 & 0.5 & 110 & ISM & 280 & $1,2,3$ \\
060926 & 3.208 & 1.0 & 8.0 & 430 & ISM & 234 & $4,2,5$ \\
080319C & 1.95 & 6.13 & 34 & 360 & ISM & 274 & 6,7 \\
080413B & 1.1 & 1.53 & 8.0 & 167 & ISM & 271 & 8,9 \\
\hline
\end{tabular}

References: 1 Cucchiara et al. (2006); 2 Butler et al. (2007); 3 Sato et al. (2006); 4 D’Elia et al. (2006); 5 Cummings et al. (2006); 6 Wiersema et al. (2008); 7 Stamatikos et al. (2008a); 8 Vreeswijk et al. (2008); 9 Barthelmy et al. (2008); ${ }^{a}$ redshift of GRB; ${ }^{b}$ isotropic equivalent energy of prompt $\gamma$-ray emission $\left(1-10^{4} \mathrm{keV}\right.$ in the burst frame for bursts with defined redshift to consistent with the catalog in Butler et al. 2007); ${ }^{c}$ time duration of GRB; ${ }^{d}$ the calculated initial Lorentz factor of fireballs (assuming $n=1 \mathrm{~cm}^{-3}$ for ISM case).

\subsection{Determining the initial Lorentz factor}

In our GRB sample, the peaks appearing in the early X-ray afterglow light curves are related to the position of the outflow $R_{\mathrm{d}}$ from the central engine. At this time, the swept medium has an energy comparable to that of the GRB outflow, and the instant Lorentz factor at $R_{\mathrm{d}}$ is about half of the initial one. Based on the assumption above, we can measure the initial Lorentz factor of GRBs in ISM case (Blandford \& Mckee 1976):

$\Gamma_{0}=\left[\frac{24 E_{\gamma}(1+z)^{3}}{\pi n m_{\mathrm{p}} c^{5} \eta t_{\mathrm{p}}^{3}}\right]^{1 / 8}$,

where $E_{\gamma}$ is the isotropic energy of prompt gamma-ray emission. Here, we take the radiation efficiency $\eta=0.2$ in the calculation according to Guetta et al. (2001) and Molinari et al. (2007). The densities $n=1 \mathrm{~cm}^{-3}$ in ISM case are assumed. Since $\Gamma_{0}$ is weakly dependent on the unknown density of the cicumburst medium in the ISM case, it can be measured relatively accurate. The results have been presented in Table 3. Usually we have $\Gamma_{0} \sim$ a few $\times 100$ in the case of ISM with a number density $n \sim 1 \mathrm{~cm}^{-3}$, consistent with some other independent probes (Sari \& Piran 1999; Lithwick \& Sari 2001; Zhang et al. 2006; Molinari et al. 2007; Jin \& Fan 2007; Pe'er et al. 2007; Zou \& Piran 2009; Zou et al. 2009), some of which are independent of the profile of the circumburst medium.

\section{Conclusion}

We used the current Swift-XRT data to constrain the profile of the circumburst medium and then measured the initial Lorentz factor of the fireballs. As a reliable probe, the X-ray light curves should have the following characters for our purpose. (i) There is a distinguished peak. (ii) Across the peak, a smooth transition to a single power-law flux is followed, and the whole profile must be consistent with the standard afterglow model. The early peaks accompanied by steep decay, usually steeper than $t^{-3}$, are abandoned. Among the $\sim 160$ Swift bursts we checked, only 4 events meet such requests, because in most events the early X-ray emission are polluted by the emission powered by the prolonged activity of the central engine. In all 4 bursts, the initial increase in the X-ray flux is quicker than $t^{2}$, strongly suggesting a constant low-density medium. In general, we find $\Gamma_{0} \sim$ a few $\times 100$, consistent with the constraints obtained in other analysis. In this work all the events in the sample are bright GRBs. For the nearby sub-luminous events, such as GRB 980425, GRB 031203, GRB 060218, and GRB 060614, a reliable estimate of their initial Lorentz factors is still infeasible at present.
In our analysis we did not find a good case for the burst born in a stellar wind and behaving as a thin shell (see Sect. 3.2). One possible reason is that usually the wind medium is so dense that the outflow has decelerated significantly on a timescale $t<T_{90}$, so the X-ray data is likely to be unsuitable as a probe of the initial Lorentz factor of GRB outflows for most of events occurring in the wind medium.

Note added in proof After the acceptance of the paper, the details of the X-ray afterglow data of GRB 090113 became available (Krimm et al. 2009): The $0.3-10 \mathrm{keV}$ light curve shows an initial period of roughly constant emission. For $t>530 \mathrm{~s}$, the light curve can be modeled with a power-law decay as $t^{-1.3}$. The $\mathrm{X}$-ray spectrum is $F_{v} \propto v^{-1.28 \pm 0.20}$. One can see that, with $p \sim 2.4$, both the temporal and the spectral behaviors of this X-ray afterglow are consistent with the forward shock emission model in the case of a wind medium and $v_{\mathrm{X}}>\max \left\{v_{\mathrm{c}}^{\mathrm{f}}, v_{\mathrm{m}}^{\mathrm{f}}\right\}$. The initial Lorentz factor can be estimated as $\Gamma_{0}=\left[\frac{2 E_{\gamma}(1+z)}{\pi A m_{\mathrm{p}} c^{3} \eta t_{\mathrm{p}}}\right]^{1 / 4}$, where $A$ is related to the regular wind parameter $A_{*}$ by $A=3 \times 10^{35} A_{*}$. Assuming $z \sim 1.0$, we have $E_{\gamma} \sim 7.9 \times 10^{51} \mathrm{erg}$ and $\Gamma_{0} \approx 121 A_{34}^{-1 / 4}$.

Acknowledgements. We thank an anonymous referee for helpful comments and Nathaniel R. Butler for providing some of the data used in this work. This work made use of data supplied by the UK Swift Science Data Centre at the University of Leicester. This work is supported by the National Science Foundation (grants 10673034 and 10621303) and National Basic Research Program (973 programs 2007CB815404 and 2009CB824800) of China. Y.Z.F. is also supported by Danish National Research Foundation and by Chinese Academy of Sciences.

\section{References}

Akerlof, C., Balsano, R., Barthelmy, S., et al. 1999, Nature, 398, 400 Bhattacharya, D. 2001, Bull. Astr. Soc. India, 29, 107

Barthelmy, S. D., Baumgartner, W., Cummings, J. R., et al. 2008, GCN Circ., 7606

Blandford, R. D., \& McKee, C. F. 1976, Phys. Fluids, 19, 1130

Butler, N. R., Kocevski, D., Bloom, J. S., \& Curtis, J. L. 2007, ApJ, 671, 656

Cheng, K. S., \& Wei, D. M. 1996, MNRAS, 283, L133

Chevalier, R. A., \& Li, Z. Y. 2000, ApJ, 536, 195

Costa, E., Frontera, F., Heise, J., et al. 1997, Nature, 387, 783

Cucchiara, A., Fox, D. B., Berger, E., \& Price, P. A. 2006, GCN Circ., 5470

Cummings, J. R., Barbier, L., Barthelmy, S. D., et al. 2006, GCN Circ., 5621

Dai, Z. G., \& Cheng, K. S. 2001, ApJ, 558, L109

D'Elia, V., Piranomonte, S., Covino, S., et al. 2006, GCN Circ., 5637

Evans, P. A., Beardmore, A. P., Page, K. L., et al. 2007, A\&A, 469, 379

Falcone, A. D., Morris, D., Racusin, J., et al. 2007, ApJ, 671, 1921

Fan, Y. Z., \& Wei, D. M. 2005, MNRAS, 364, L42

Fan, Y. Z., Dai, Z. G., Huang, Y. F., \& Lu, T. 2002, ChJAA, 2, 449

Gallant, Y. A. 2002, Lecture Notes in Physics, 589, 24

Gehrels, N., Chincarini, G., Giommi, P., et al. 2004, ApJ, 611, 1005 
Guetta, D., Spada, M., \& Waxman, E. 2001, ApJ, 557, 399

Holland, S. T., Barthelmy, S. D., Starling, R., et al. 2006, GCN Rep., 1.2

Holland, S. T., Palmer, D. M., Starling, R. L. C., \& Schady, P. 2008, GCN Rep.,

128.1

Jin, Z. P., \& Fan, Y. Z. 2007, MNRAS, 378, 1043

Klebesadel, R. W., Strong, I. B., \& Olson, R. A. 1973, ApJ, 182, L85

Kobayashi, S. 2000, ApJ, 545, 807

Krimm, H. A., Evans, P. A., Oates, S. R., et al. 2009, GCN Rep., 193.1

Lithwick, Y., \& Sari, R. 2001, ApJ, 555, 540

Mészáros, P., \& Rees, M. J. 1999, MNRAS, 306, L39

Molinari, E., Vergani, S. D., Malesani, D., et al. 2007, A\&A, 469, L13

Oates, S. R., et al. 2009, MNRAS, in press [arXiv: 0901.3597]

Pagani, C., Racusin, J. L., Kennea, J. A., et al. 2008, GCN Circ, 7460

Page, K. L., Osborne, J. P., \& Holland, S. T. 2008, GCN Circ, 7376

Pe'er, A., Ryde, F., Wijers, R. A. M. J., Mészáros, P., \& Rees, M. J. 2007, ApJ, 664, L1

Piran, T., Shemi, A., \& Narayan, R. 1993, MNRAS, 263, 861

Racusin, J. L., Barbier, L. M., Brown, P. J., et al. 2006a, GCN Circ., 5378

Racusin, J. L., Grupe, D., Morris, D., \& Stroh, M. 2006b, GCN Circ., 5382
Rhoads, J. E. 1999, ApJ, 525, 737

Sari, R., \& Piran, T. 1999, ApJ, 520, 641

Sari, R., Piran, T., \& Narayan, R. 1998, ApJ, 497, L17

Sato, G., Barbier, L., Barthelmy, S. D., et al. 2006, GCN Circ., 5381

Shemi, A., \& Piran, T. 1990, ApJ, 365, L55

Stamatikos, M., Barthelmy, S. D., Cummings, J., et al. 2008a, GCN Circ., 7483

Stamatikos, M., Beardmore, A. P., Gehrels, N., et al. 2008b, GCN Circ., 7598

Troja, E., \& Stamatikos, M. 2008, GCN Circ., 7608

Vreeswijk, P. M., Thoene, C. C., Malesani, D., et al. 2008, GCN Circ., 7601

Waxman, E. 1997, ApJ, 485, L5

Wei, D. M. 2007, MNRAS, 374, 525

Wiersema, K., Tanvir, N., Vreeswijk, P., et al. 2008, GCN Circ., 7517

Wijers, R. A., Rees, M. J., \& Mészáros, P. 1997, MNRAS, 288, L51

Zhang, B. 2007, ChJAA, 7, 1

Zhang, B., Fan, Y. Z., Dyks, J., et al. 2006, ApJ, 642, 354

Zou, Y. C., \& Piran, T. 2009, in preparation

Zou, Y. C., Wu, X. F., \& Dai, Z. G. 2005, MNRAS, 363, 93

Zou, Y. C., Fan, Y. Z., \& Piran, T. 2009, MNRAS, submitted [arXiv:0811.2997] 